\title{
Schistosomal Peritonitis Presenting as Acute Abdomen: A Case Report
}

\author{
Kirubel Abebe $^{{ }^{*}}$, Abebe Megersa ${ }^{1}$, Engida Abebe $^{1}$
}

\section{OPEN ACCESS}

Citation: Kirubel Abebe, Abebe Megersa, Engida Abebe. Schistosomal peritonitis presenting as acute abdomen: A case report. Ethiop J Health Sci. 2019; 29(6):783.doi:http://dx.doi.org/10.4314/ej hs.v29 i6.17

Received: July 21, 2019

Accepted: August 01, 2019

Published: November 1, 2019

Copyright: (C2019 Kirubel A, et al. This is an open access article distributed under the terms of the Creative Commons Attribution License, which permits unrestricted use, distribution, and reproduction in any medium, provided the original author and source are credited. Funding: Nil

Competing Interests: The authors declare that this manuscript was approved by all authors in its form and that no competing interest exists.

Affiliation and Correspondence:

${ }^{1}$ Department of Surgery, St. Paul's

Hospital Millennium Medical College,

Addis Ababa, Ethiopia

*Email:kirumel@yahoo.com

\begin{abstract}
Background-Schistosomiasis is a trematode infestation causing a chronic granulomatous disease in various organs. Both $S$. mansoni \& S. haematobium are endemic in Ethiopia. Most infected individuals are asymptomatic. Ectopic schistosomiasis can affect the lungs, genitalia, CNS, skin, peritoneum, Lymph nodes \& other organs. Schistosomiasis as a cause of acute abdomen is seldom reported.

Case Detail-A 51years -old male Ethiopian farmer presented with a two weeks history of abdominal pain with recent onset bilious vomiting and abdominal distention. Emergency laparotomy done \& the finding was multiple tiny whitish nodule over the peritoneum \& small bowel with multiple mesenteric lymphadenopathy. The diagnosis was confirmed with histopathology study.

Conclusions- Schistosomal peritonitis is a very uncommon form of schistosomiasis. Physicians should be aware of such atypical presentation in patients from endemic areas of schistosomiasis. And biopsy should be considered in unsettled forms of peritonitis during laparotomy. The pathogenesis is not well known which warrants further study.
\end{abstract}

Key words- Peritoneal schistosomiasis, acute abdomen, S. mansoni

\section{INTRODUCTION}

Schistosomiasis is a trematode infestation causing both acute disease and chronic granulomatous disease in various organs (1). Around 240 million people are infected worldwide \& 97\% of these Infections occur in sub-Sahara African countries. It is acquired through Contact with fresh water contaminated with the larval form. Urogenital schistosomiasis is caused by Schistosoma haematobium and intestinal Schistosomiasis by S. mansoni, most commonly. But S. guineensis, S. intercalatum, S. japonicum, or S. mekongi can cause intestinal Schistosomiasis (1).

In Ethiopia, both S. mansoni \& S. haematobium are endemic. The prevalence of S. mansoni ranges from 10 to $90 \%$. Agricultural communities along streams between 1300 and $2000 \mathrm{~m}$ altitude are commonly affected (2). Most infected individuals do not develop symptoms. When seen symptoms of intestinal schistosomiasis are nonspecific and includes chronic or intermittent abdominal pain, 
diarrhea with or without blood \& fever. Ectopic schistosomiasis can affect the lungs, genitalia, CNS, skin, peritoneum, Lymph nodes \& other organs (1). Schistosomiasis as a cause of acute abdomen is seldom reported

Here we report 51 years old male patient presented with symptoms suggestive of gangrenous small bowel obstruction and later diagnosed as peritoneal and mesenteric lymph node Schistosomiasis by histopathology. The challenges in making the diagnosis is discussed.

\section{THE CASE}

A 50 years-old male farmer from southwestern part of Ethiopia presented with a 2 weeks history of intermittent abdominal pain associated with fever \& bloody diarrhea. The pain became more severe, persistent \& diffuses in the past 3 days prior to presentation. The patient also developed frequent bilious vomiting, abdominal distention \& failure to pass feces and flatus of the same duration.

On examination, he was ill-looking, dehydrated \& tachycardic (120 bpm). Abdomen was distended with generalized direct $\&$ rebound tenderness. Bowel sound was absent \& digital rectal examination revealed only blood on examining finger. No abnormality detected in other systems.
Lab blood tests showed normal Hemoglobin $\&$ renal function tests. But he had Leukocytosis $(14,000)$ and differential count showed 62\% neutrophils and $9 \%$ eosinophils. Erect Plain abdominal X-ray finding was consistent with sign of small bowel obstruction.

Patient was prepared for urgent laparotomy with presumptive diagnosis of gangrenous small bowel obstruction. The exploration showed multiple tiny whitish nodule over the peritoneum \& small bowel (mainly over the terminal ileum). There were also edematous dilated small bowel loops, multiple mesenteric lymphadenopathy \& minimal ascites. Other intra-abdominal organs including the appendix were grossly normal. Patency of bowel confirmed, lymph node biopsy taken, abdomen washed \& closed. The possibility of Tuberculose peritonitis considered and patient started empirically on anti-Tuberculosis (anti-TB) till biopsy result collected. Later, histopathologic examination revealed Schistosoma ova having Spica which confirm peritoneal (mesenteric lymph node) schistosomiasis (figure 1). With this diagnosis the anti-TB was discontinued \& patient treated with Praziquantel (4omg/Kg stat dose). On subsequent follow ups patient showed significant improvement and had smooth post operative and post treatment course.

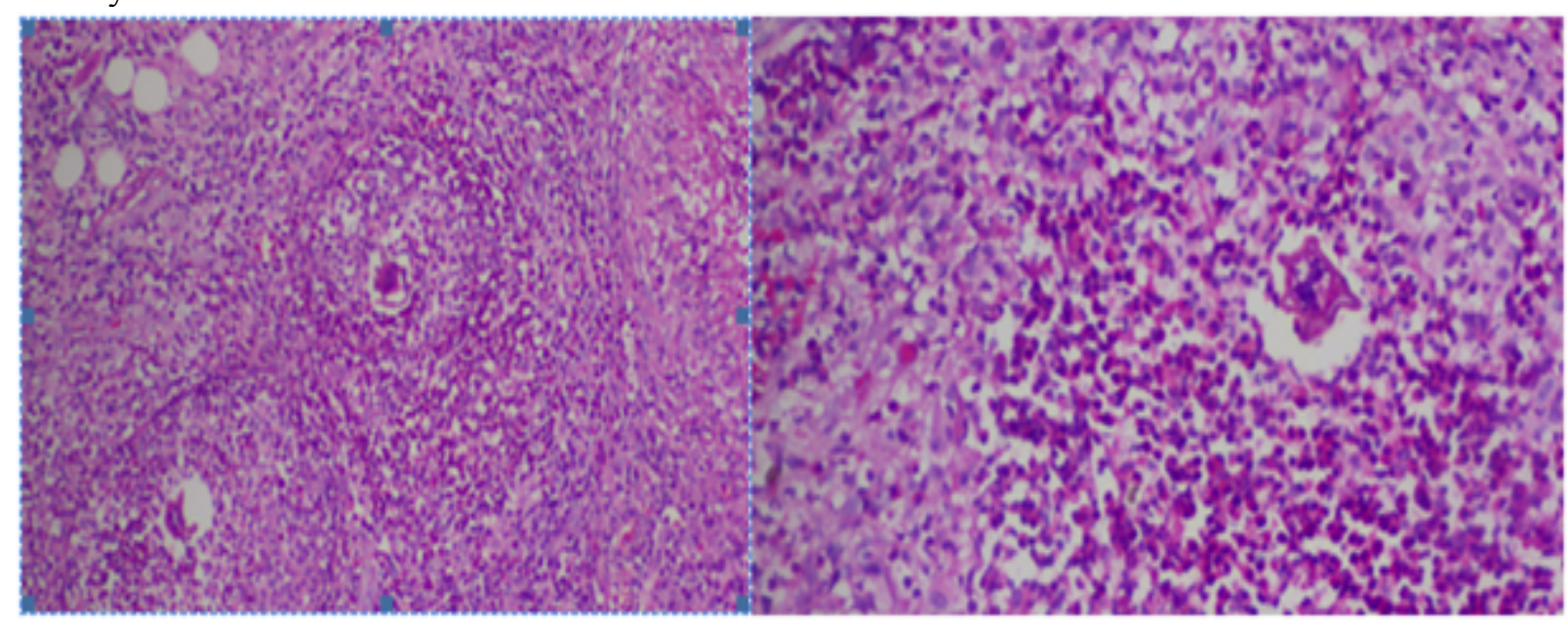

Figure1: low power field (Left) and high-power field (right) magnification of lymph node showing Largely effaced architecture with mixed inflammatory cell population and frequent intravascular and dispersed Schistosoma ova having Spica with surrounding epithelioid granuloma, fibrosis and extensive eosinophilia

DOI: http://dx.doi.org/10.4314/ejhs.v29i6.17 


\section{DISCISSION}

Human schistosomiasis commonly affects colon, rectum \& urinary bladder (1). However, Ectopic or unusual sites such as appendix, lungs, genitalia, CNS, skin \& peritoneum are reported $(1,3,4)$. Aldossary, et al. reported peritonitis due to histologically confirmed perforated Schistosomal appendicitis in a Tanzanian patient (3).

In non -endemic areas immigrants can present with acute peritonitis as it is reported by $\mathrm{E}$ Konstantinidou et al in Egyptian immigrant. Histopathology study found eggs of Schistosoma mansoni in the greater omentum \& peritoneum (4). Involvement of other organs such as the small intestine $\&$ rectum can present with perforation $\&$ peritonitis (5).

The exact mechanism of peritoneal Schistosomiasis is not known. The inflammatory adhesion \& intramuscular oviposition causing obstruction then perforation is mentioned as a proposed theory. Others believe that eggs emboli to the mesenteric veins causing bowel ischemia then perforation (3). In contrast to the above theories, our patient has no viscus perforation which might indicate the possibility of other mechanism such as lymphatic spread.

Due to the absence of specific feature of schistosomiasis, diagnosis relies on histopathologic study of the affected organ or Abdominal ultrasound in case of liver schistosomiasis in endemic areas. The presented case shows that peritoneal schistosomiasis can be confused with other causes of acute peritonitis. The additional finding in our patient is the involvement of the mesenteric lymph nodes in the process. This is a very uncommon form of schistosomiasis which warrants further study in the pathogenesis of the diseases. In general, Physicians should be aware of such atypical presentation in patients from endemic areas of schistosomiasis. And biopsy should be considered in unsettled forms of peritonitis during laparotomy. Praziquantel $40 \mathrm{mg} / \mathrm{kg}$ is the standard treatment for schistosomiasis caused by $\mathrm{S}$. mansoni or S. japonicum $(1,3)$.

\section{REFERENCES}

1. Gryseels B, Polman K, Clerinx J, Kestens L. Human schistosomiasis. The Lancet. 2006 Sep 23;368(9541):1106-18.

2. Kloos H, Lo CT, Birrie H, Ayele T, Tedla S, Tsegay F. Schistosomiasis in Ethiopia. Social Science \& Medicine. 1988 Jan 1;26(8):803-27.

3. Aldossary MY, Almabyouq F, Mashhour M, Hassan K. Schistosomal appendicitis presenting as acute peritonitis: A case report and literature review. Journal of Health Specialties. 2017 Oct 1;5(4):225.

4. Konstantinidou E, Alexiou C, Demonakou M, Sakellaridis T, Fotopoulos A, Antsaklis G. Schistosomal peritonitis: a rare cause of acute abdomen. Transactions of the Royal Society of Tropical Medicine and Hygiene. 2009 Oct 1;103(10):1068-70.

5. Xiang JJ, Cheng BJ, Tian F, Li M, Jiang XF, Zhao HC, Hu XM, Xiao BL, Xie JP, Shrestha A. Perforation of small bowel caused by Schistosoma japonicum: A case report. World Journal of Gastroenterology: WJG. 2015 Mar 7;21(9):2862. 